\title{
Cold nuclear matter effects on $J / \psi$ production: intrinsic and extrinsic transverse momentum effects
}

\author{
E. G. Ferreiro ${ }^{\mathrm{a}}$, F. Fleuret ${ }^{\mathrm{b}}$, J.P. Lansberg ${ }^{\mathrm{c}, \mathrm{d}, 1}$, A. Rakotozafindrabe ${ }^{\mathrm{e}}$, \\ ${ }^{a}$ Departamento de Física de Partículas, Universidad de Santiago de Compostela, 15782 Santiago de Compostela, Spain \\ ${ }^{b}$ Laboratoire Leprince Ringuet, École Polytechnique, CNRS-IN2P3, 91128 Palaiseau, France \\ ${ }^{c}$ SLAC National Accelerator Laboratory, Theoretical Physics, Stanford University, Menlo Park, CA 95025, USA \\ ${ }^{d}$ Institut für Theoretische Physik, Universität Heidelberg, Philosophenweg 19, D-69120 Heidelberg, Germany \\ ${ }^{e}$ IRFU/SPhN, CEA Saclay, 91191 Gif-sur-Yvette Cedex, France
}

\begin{abstract}
Cold nuclear matter effects on $J / \psi$ production in proton-nucleus and nucleus-nucleus collisions are evaluated taking into account the specific $J / \psi$-production kinematics at the partonic level, the shadowing of the initial parton distributions and the absorption in the nuclear matter. We consider two different parton processes for the $c \bar{c}$-pair production: one with collinear gluons and a recoiling gluon in the final state and the other with initial gluons carrying intrinsic transverse momentum. Our results are compared to RHIC observables. The smaller values of the nuclear modification factor $R_{A A}$ in the forward rapidity region (with respect to the mid rapidity region) are partially explained, therefore potentially reducing the need for recombination effects.
\end{abstract}

Key words: $J / \psi$ production, heavy-ion collisions, cold nuclear matter effects

\section{Introduction}

The charmonium production in hadron collisions is an on-going major subject of investigations, on both experimental and theoretical sides. It has been widely studied in $p p$ collisions; our understanding was recently reviewed in $[1,2]$. It may also be used as a tool to probe the medium produced in nucleus-nucleus $(A B)$ collisions (for a recent review, see [3] along with some perspectives for the LHC [4]). This medium is expected to be in a deconfined state of QCD matter - such as the Quark Gluon Plasma (QGP) - at high enough temperatures and densities. The $J / \psi$ production should be sensitive to the QGP formation, due to competing effects such as a color Debye screening suppression [5] or the so-called recombination mechanism [6]. Recent results on $J / \psi$ production are available from the PHENIX experiment at the BNL Relativistic Heavy Ion Collider (RHIC). They show a significant suppression of the $J / \psi$ yield in AuAu collisions at $\sqrt{s_{N N}}=200 \mathrm{GeV}$ [7] compared to the expected yield from $p p$ measurements [8]. However, the interpretation relies on a good understanding and a proper subtraction of the Cold Nuclear Matter (CNM) effects, known to impact the $J / \psi$ production in proton(deuteron)-nucleus $(p A, d A)$ collisions where the deconfinement can not be reached. Indeed, the $p A$ data [9] obtained at the SPS energies can be described by assuming the break-up of the pre-resonant $c \bar{c}$ pair due to multiple scattering along its way to escape the nuclear environment - the so-called nuclear absorption. PHENIX data

\footnotetext{
${ }^{1}$ Present address at SLAC

on $d \mathrm{Au}$ collisions [10] have also revealed that CNM effects play an essential role at RHIC energy. These effects a priori include shadowing, i.e. the modification of the parton distribution of a nucleon in a nucleus, and final-state nuclear absorption.

As we shall show thereafter, the impact of gluon shadowing depends on the partonic process producing the $c \bar{c}$ and then the $J / \psi$. So far, the studies of $J / \psi$ production $[11,12,13]$ with gluon shadowing relied on the assumption that the $c \bar{c}$ pair was produced by the fusion of two gluons carrying some intrinsic transverse momentum $k_{T}$. The partonic process being a $2 \rightarrow 1$ scattering, the sum of the gluon intrinsic transverse momentum is transferred to the $c \bar{c}$ pair, thus to the $J / \psi$ since the soft hadronisation process does not modify the kinematics. This corresponds to the picture of the Colour Evaporation Model (CEM) at LO (see [1] and references therein).

In such approaches, the transverse momentum of the $J / \psi$ entirely comes from the intrinsic transverse momentum of the initial gluons. This seems acceptable for the low- $P_{T}$ region and the origin of this intrinsic $P_{T}$ can be paralleled to the increase of $\left\langle P_{T}^{2}\right\rangle$ when going from $p p$ to $p A$ and for increasing atomic number $A$. This is known as the Cronin effect: the increase of $\left\langle P_{T}^{2}\right\rangle$ is believed to come from a broadening of the intrinsic transverse momentum distribution, resulting from the multiple scatterings experienced by the initial gluon from the proton as it goes through the target nucleus before the heavy-quark production [14].

However, such an effect is not sufficient to describe the $P_{T}$ spectrum of quarkonia produced in hadron collisions [1]. Most of the transverse momentum should have an extrinsic

July 30, 2009 
origin, i.e. the $J / \psi$ 's $P_{T}$ would be balanced by the emission of a recoiling particle in the final state. The $J / \psi$ would then be produced by gluon fusion and with emission of a hard final-state gluon. For the production of ${ }^{3} S_{1}$ states - like the $J / \psi$, such a $2 \rightarrow 2$ partonic process is anyhow mandatory to satisfy $C$-parity conservation.

It is among our purposes here to investigate the influence of such an emission on the kinematics of the $J / \psi$ production in $p(d) A$ and $A A$ collisions. Indeed, for a given $J / \psi$ momentum (thus for fixed $y$ and $P_{T}$ ), the processes discussed above, i.e. $g+g \rightarrow c \bar{c} \rightarrow J / \psi(+X)$ and $g+g \rightarrow J / \psi+g$, will proceed on the average from gluons with different Bjorken$x$. Therefore, they will be affected by different shadowing corrections. From now on, we will refer to the former scenario as the intrinsic scheme, and to the latter as the extrinsic scheme. In the following, we shall consider them as distinct approaches ${ }^{2}$.

In practice, we shall study the kinematic regime at RHIC at $\sqrt{s_{N N}}=200 \mathrm{GeV}$. For the extrinsic scheme, we shall consider the partonic differential cross section for $g+g \rightarrow$ $J / \psi+g$ given in [15] which satisfactorily describes the data obtained in $p p$ collisions at RHIC down to $P_{T} \sim 0$ (see Fig. 1). For the intrinsic scheme, we shall follow the studies $[11,12,13]$ based on $(2 \rightarrow 1)$-like processes where the momentum of the particles denoted by $X$ in $g+g \rightarrow$ $c \bar{c} \rightarrow J / \psi(+X)$ is neglected. Using a probabilistic Glauber Monte-Carlo code, we interface these production processes with CNM effects, such as shadowing and nuclear absorption, in order to get the $J / \psi$ production cross sections for $p A$ and $A A$ collisions. We shall finally compare our results with the experimental measurements presently available at RHIC.

\section{The Monte-Carlo framework for $J / \psi$ production}

To describe the $J / \psi$ production in nucleus collisions, our Monte-Carlo framework is based on the probabilistic Glauber model, the nuclear density profiles being defined with the Woods-Saxon parameterisation for any nucleus $A>2$ and the Hulthen wavefunction for the deuteron [17]. The nucleon-nucleon inelastic cross section at $\sqrt{s_{N N}}=$ $200 \mathrm{GeV}$ is taken to $\sigma_{N N}=42 \mathrm{mb}$ and the maximal nucleon density to $\rho_{0}=0.17$ nucleons $/ \mathrm{fm}^{3}$. For each event (for each $A B$ collision) at a random impact parameter $b$, the Glauber Monte-Carlo model allows us to determine the number of nucleons in the path of each incoming nucleon, therefore allowing us to easily derive the number $N_{\text {coll }}$ of nucleonnucleon collisions and the total number $N_{\text {part }}$ of nucleons participating into the collision.

\footnotetext{
${ }^{2}$ In the extrinsic scheme and for this first study, we shall neglect the small kinematical effects of the yield from the decay of $\chi_{c}$ produced by $2 \rightarrow 1$ processes. Indeed, from the $25 \%$ of the $\chi_{c}$ feeddown [16], it is reasonable to suppose that only half of it effectively proceeds via a $g g \rightarrow$ $\chi_{c}$ which is allowed at LO in $\alpha_{S}$ only for $\chi_{c 2}$, not for $\chi_{c 1}$.
}

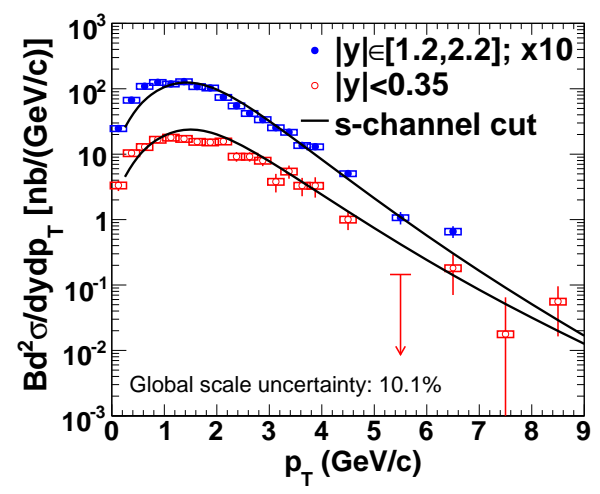

(a) $P_{T}$ distributions from the $s$-channel cut contributions compared to PHENIX $p p$ data [8] in the forward and central rapidity regions.

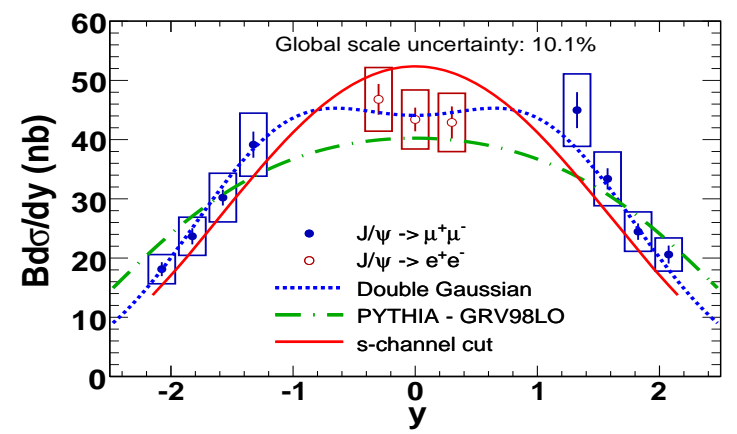

(b) Rapidity spectrum from $s$-channel cut contributions compared to PHENIX $p p$ data, the ad hoc double-Gaussian fit [8] and the predictions from the PYTHIA event generator.

Figure 1: $P_{T}$ and $y$ spectra in $p p$ at $\sqrt{s_{N N}}=200 \mathrm{GeV}$.

\subsection{The CNM effects}

To get the $J / \psi$ yield in $p A$ and $A A$ collisions, a shadowing-correction factor has to be applied to the $J / \psi$ yield obtained from the simple superposition of the equivalent number of $p p$ collisions. This shadowing factor can be expressed in terms of the ratios $R_{i}^{A}$ of the nuclear Parton Distribution Functions (nPDF) in a nucleon of a nucleus $A$ to the PDF in the free nucleon. In the following, we will consider the evolution model EKS98 [18] which is recognised to be a reasonable compromise between DS [19] and EPS08 [20] as regards the strength of the gluon antishadowing for instance. It provides $R_{i}^{A}$ at a given initial value of $\mu_{F}$ - the factorisation scale - and takes into account their evolution through the DGLAP equations. The nuclear ratios of the PDFs are expressed by:

$$
R_{i}^{A}\left(x, \mu_{F}\right)=\frac{f_{i}^{A}\left(x, \mu_{F}\right)}{A f_{i}^{\text {nucleon }}\left(x, \mu_{F}\right)}, f_{i}=q, \bar{q}, g .
$$

Within EKS98, these nuclear ratios are parameterized at some initial scale $\mu_{F, 0}^{2}=2.25 \mathrm{GeV}^{2}$ which is assumed large enough for perturbative DGLAP evolution to be applied. They are evolved at LO from $\mu_{F, 0}^{2}$ up to $\mu_{F}^{2}\left(<10^{4} \mathrm{GeV}^{2}\right)$ and are valid for $x \geq 10^{-6}$. The numerical parameterisation of $R_{i}^{A}\left(x, \mu_{F}\right)$ is given for all parton flavours. Here, we 
restrain our study to gluons since, at high energy, $J / \psi$ is essentially produced through gluon fusion [1]. Usually, the spatial dependence of $R_{i}^{A}\left(x, \mu_{F}\right)$ is not given. However, as we shall see in the next section, it can be included in our approach.

The second CNM effect that we are going to take into account concerns the nuclear absorption. In the framework of the probabilistic Glauber model, this effect refers to the probability for the pre-resonant $c \bar{c}$ pair to survive to the propagation through the nuclear medium and is usually parametrised by introducing an effective absorption cross section $\sigma_{\text {abs }}$.

\subsection{The differential $J / \psi$-production cross section in AB collisions}

Our Glauber Monte-Carlo framework is aimed at numerically evaluating the differential $J / \psi$-production cross section in nucleus collisions, by exploring the whole physical phase space with a proper weighting of each point in this phase space. Within this Glauber-based calculation, the differential cross section for the production (via gluon fusion) of a quarkonium with momentum $\left(y, P_{T}\right)$, in nucleus collisions at impact parameter $\vec{b}$ and for a nucleon-nucleon CM energy of $\sqrt{s_{N N}}$ can be represented by a generic integral. It takes two different forms depending on the kinematics of the partonic process responsible for the $J / \psi$ production.

The intrinsic scheme corresponds to a $2 \rightarrow 1$ partonic process, with initial gluons carrying a non-zero intrinsic transverse momentum. Following [13], we do not neglect the value of the $J / \psi$ 's $P_{T}$ in this simplified kinematics. In this scheme, the measurement of the $J / \psi$ momentum completely fixes the longitudinal momentum fraction carried by the initial partons:

$$
x_{1,2}=\frac{m_{T}}{\sqrt{s_{N N}}} \exp ( \pm y) \equiv x_{1,2}^{0}\left(y, P_{T}\right)
$$

with the transverse mass $m_{T}=\sqrt{M^{2}+P_{T}^{2}}, M$ being the $J / \psi$ mass.

Therefore, we can write

$$
\begin{aligned}
& \frac{d \sigma_{A B}^{\text {Intr. }}}{d y d P_{T} d \vec{b}}=\int d \vec{r}_{A} d z_{A} d z_{B} \mathcal{F}_{g}^{A}\left(x_{1}^{0}, \vec{r}_{A}, z_{A}, \mu_{F}\right) \\
& \mathcal{F}_{g}^{B}\left(x_{2}^{0}, \vec{r}_{B}, z_{B}, \mu_{F}\right) \sigma_{g g}^{\text {Intr. }}\left(x_{1}^{0}, x_{2}^{0}\right) S_{A}\left(\vec{r}_{A}, z_{A}\right) S_{B}\left(\vec{r}_{B}, z_{B}\right)
\end{aligned}
$$

with

- $\vec{r}_{A}\left[\vec{r}_{B}=\vec{b}-\vec{r}_{A}\right]$ and $z_{A}\left[z_{B}\right]$ are the transverse and longitudinal spatial locations of the initial parton (a gluon here) in the nucleus $A[B]$; it carries the longitudinal momentum fraction $x_{1}^{0}\left[x_{2}^{0}\right]$ and can be found with the probability $\mathcal{F}_{g}^{A}\left[\mathcal{F}_{g}^{B}\right]$ at a scale $\mu_{F}$;

- The nuclear absorption is taken into account through $S_{A}\left(\vec{r}_{A}, z_{A}\right)=\exp \left(-A \sigma_{\text {abs }} \int_{z_{A}}^{\infty} d \tilde{z} \rho_{A}\left(\vec{r}_{A}, \tilde{z}\right)\right)$ $\left[S_{B}\left(\vec{r}_{B}, z_{B}\right)\right]$, which stands for the survival probability for a $c \bar{c}$ produced at the point $\left(\vec{r}_{A}, z_{A}\right)\left[\left(\vec{r}_{B}, z_{B}\right)\right]$ to pass through the projectile and the target unscathed;
- $\sigma_{g g}^{\text {Intr. }}\left(x_{1}^{0}, x_{2}^{0}\right)$ is the partonic cross section for the process $g+g \rightarrow c \bar{c} \rightarrow J / \psi(+X)$ and is a function of $P_{T}$ and $y$ through $x_{1}^{0}$ and $x_{2}^{0}$. As we will show in the section 2.3, it can be extracted from experimental data along with the PDFs.

In the extrinsic scheme, we deal with a $2 \rightarrow 2$ partonic process with collinear initial gluons and we have

$$
\begin{aligned}
& \frac{d \sigma_{A B \rightarrow J / \psi X}}{d y d P_{T} d \vec{b}}=\int d x_{1} d x_{2} \int d \vec{r}_{A} d z_{A} d z_{B} \mathcal{F}_{g}^{A}\left(x_{1}, \vec{r}_{A}, z_{A}, \mu_{F}\right) \\
& \mathcal{F}_{g}^{B}\left(x_{2}, \vec{r}_{B}, z_{B}, \mu_{F}\right) 2 \hat{s} P_{T} \frac{d \sigma_{g g \rightarrow J / \psi+g}}{d \hat{t}} \delta\left(\hat{s}-\hat{t}-\hat{u}-M^{2}\right) \\
& S_{A}\left(\vec{r}, z_{A}\right) S_{B}\left(\vec{r}_{B}, z_{B}\right),
\end{aligned}
$$

with

- $\hat{s}=s_{N N} x_{1} x_{2}, \hat{t}=M^{2}-x_{1} \sqrt{s_{N N}} m_{T} e^{y}, \hat{u}=M^{2}-$ $x_{2} \sqrt{s_{N N}} m_{T} e^{-y}$. The four-momentum conservation - represented by the $\delta$ function - explicitly results in a more complex expression of $x_{2}$ as a function of $\left(x_{1}, y, P_{T}\right)$ :

$$
x_{2}=\frac{x_{1} m_{T} \sqrt{s_{N N}} e^{-y}-M^{2}}{\sqrt{s_{N N}}\left(\sqrt{s_{N N}} x_{1}-m_{T} e^{y}\right)} .
$$

Equivalently, a similar expression can be written for $x_{1}$ as a function of $\left(x_{2}, y, P_{T}\right)$;

- $d \sigma_{g g \rightarrow J / \psi+g} / d \hat{t}$ can be computed in a priori different approaches that correctly describes the $p p$ data. For now, we use the one obtained in [15], but others can be interfaced with our code. See section 2.3 for further details.

Now, concerning $\mathcal{F}_{g}^{A}\left(x_{1}, \vec{r}_{A}, z_{A}, \mu_{F}\right)$, we assume that it can be factorised in the nuclear density distribution $\rho_{A}\left(\vec{r}_{A}, z_{A}\right)$, the shadowing modification factor $\mathcal{R}_{g}^{A}\left(\vec{r}_{A}, x, \mu_{F}\right)$ and the usual gluon PDFs $g\left(x ; \mu_{F}\right)$ :

$$
\mathcal{F}_{g}^{A}\left(x_{1}, \vec{r}_{A}, z_{A} ; \mu_{F}\right)=\rho_{A}\left(\vec{r}_{A}, z_{A}\right) \mathcal{R}_{g}^{A}\left(\vec{r}_{A}, x_{1}, \mu_{F}\right) g\left(x_{1} ; \mu_{F}\right) .
$$

A priori, the modifications of the nPDFs should depend on the parton position $(\vec{r}, z)$ in the nucleus. Such information is not experimentally available. So the centrality dependence is not encoded in the EKS98 parametrisation. However, some approaches provide an Ansatz for such a dependence. Assuming that the inhomogeneous shadowing is proportional to the path length $[21,12]$, then

$$
\mathcal{R}_{g}^{A}\left(\vec{r}_{A}, x, \mu_{F}\right)=1+\left[R_{g}^{A}\left(x, \mu_{F}\right)-1\right] N_{\rho_{A}} \frac{\int d z \rho_{A}\left(\vec{r}_{A}, z\right)}{\int d z \rho_{A}(0, z)}
$$

where $R_{g}^{A}\left(x, \mu_{F}\right)$ is the ratio nPDF/PDF given by the EKS98 parametrisation for the gluon (see Eq. (1)) and $N_{\rho_{A}}$ is a normalisation factor, determined such that

$$
\frac{1}{A} \int d^{2} \vec{r}_{A} \int d z_{A} \rho_{A}\left(\vec{r}_{A}, z_{A}\right) \mathcal{R}_{g}^{A}\left(\vec{r}_{A}, x, \mu_{F}\right)=R_{g}^{A}\left(x, \mu_{F}\right) .
$$


The integral over $z$ in Eq. (6) includes all the material traversed by the incident nucleon. This amounts to consider the incident parton as coherently interacting with all the target partons along its path length.

In the following, we shall set the scale $\mu_{F}$ in $\mathcal{F}_{g}^{A}$ equal to the renormalisation scale $\mu_{R}$ of the partonic process and take the usual choice $\mu_{R}=m_{T}, m_{T}$ providing a typical scale of the partonic process.

\subsection{The partonic cross sections}

Within the intrinsic scheme, the measurement of the differential cross section in $p p$ on Fig. 1 (a) and (b) directly provides us with values for the product of the PDFs and the partonic cross section in Eq. (3). Indeed,

$$
\frac{d \sigma_{p p}^{\text {Intr. }}}{d y d P_{T}}=g\left(x_{1}^{0} ; m_{T}\right) g\left(x_{2}^{0} ; m_{T}\right) \sigma_{g g}^{\text {Intr. }}\left(x_{1}^{0}, x_{2}^{0}\right) \text {. }
$$

In order to evaluate the integral Eq. (3), we randomly pick $y$ and $P_{T}$ out of their respective distributions obtained from fits to the experimental $p p$ spectra $d \sigma_{p p}^{\text {Intr. }} / d y d P_{T}$. For the present study, we use the fits to the $y^{3}$ and $P_{T}$ spectra measured by PHENIX [8] in $p p$ collisions at $\sqrt{s_{N N}}=200 \mathrm{GeV}$ as inputs of the Monte-Carlo. The azimuthal-angle $\varphi$ of $P_{T}$ in the $\left(P_{x}, P_{y}\right)$ plane is also random and follows a flat distribution within $[0,2 \pi]$. As discussed previously, the knowledge of $\left(y, P_{T}\right)$ unequivocally fixes the other variables $\left(x_{1}\right.$, $x_{2}$ and $\mu_{F}=m_{T}$ ) needed to compute the shadowing correction factors.

On the other hand, in the extrinsic scheme, information from the data alone - the $y$ and $P_{T}$ spectra - is not sufficient to determine $x_{1}$ and $x_{2}$. Indeed, the presence of a final-state gluon authorises much more freedom to choose $\left(x_{1}, x_{2}\right)$ for a given set $\left(y, P_{T}\right)$. Even if kinematics determine the physical phase space, models are anyhow mandatory to compute the proper weighting of each kinematically allowed $\left(x_{1}, x_{2}\right)$. This weight is simply the differential cross section at the partonic level times the gluon PDFs, i.e. $g\left(x_{1}, \mu_{F}\right) g\left(x_{2}, \mu_{F}\right) d \sigma_{g g \rightarrow J / \psi+g} / d y d P_{T} d x_{1} d x_{2}$. In the present implementation of our code, we are able to use the partonic differential cross section computed from any theoretical approach. For now, we use the one from [15] which takes into account the $s$-channel cut contributions [22] to the basic Color Singlet Model (CSM) [23] and satisfactorily describes the data down to very low $P_{T}$, where the bulk of the cross section lies. As shown on Fig. 1, this approach ${ }^{4}$ gives a fairly good description of both the $P_{T}$ and $y$ dependence of the $p p$ data at RHIC.

To evaluate the integral in the extrinsic scheme, we also randomly pick $y$ and $P_{T}$, but out of the distributions computed with the cross section computed as in [15]. For a given set $\left(y, P_{T}\right)$, the set $\left(x_{1}, x_{2}\right)$ is randomly chosen in its kinematically allowed range and follows the distribution $g\left(x_{1}, \mu_{F}\right) g\left(x_{2}, \mu_{F}\right) d \sigma_{g g \rightarrow J / \psi+g} / d y d P_{T} d x_{1} d x_{2}$.

\footnotetext{
${ }^{3}$ The double gaussian parametrisation as quoted in [8]

${ }^{4}$ The determination of the two parameters of this approach [15] has been improved by fitting RHIC $p p$ data $(a=3.2$ and $\kappa=6.3 \mathrm{GeV})$.
}

\subsection{Picturing intrinsic vs extrinsic scheme}

Fig. 2 shows the physical phase space in the $\left(x_{2}, y\right)$ plane for both schemes in $d$ Au collisions. At a fixed value of $y$, they give quite different distributions of the Bjorken- $x$ of the initial gluons. The momentum of the final-state gluon considered in the extrinsic scheme results in a larger $x_{2}$ range, whereas the intrinsic scheme much heavily favours a tighter band at low $x_{2}$.
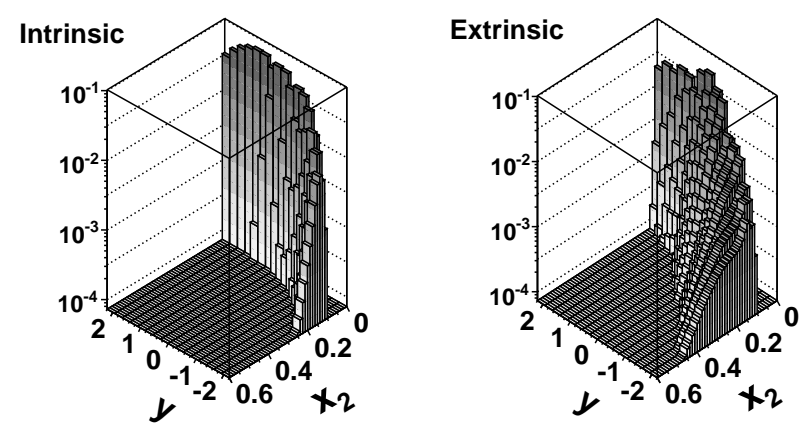

Figure 2: Normalised $x_{2}$ distribution versus rapidity in the intrinsic and extrinsic scenarios for similar Monte-Carlo $J / \psi$ statistics in $d$ Au collisions at $\sqrt{s_{N N}}=200 \mathrm{GeV}$.

\section{Results}

In the following, we present our results for the $J / \psi$ nuclear modification factor:

$$
R_{A B}=\frac{d N_{A B}^{J / \psi}}{\left\langle N_{\text {coll }}\right\rangle d N_{p p}^{J / \psi}} .
$$

$d N_{A B}^{J / \psi}\left(d N_{p p}^{J / \psi}\right)$ is the $J / \psi$ yield observed in $A B(p p)$ collisions and $\left\langle N_{\text {coll }}\right\rangle$ is the average number of nucleon-nucleon collisions occurring in one $A B$ collision. In the absence of nuclear effects, $R_{A B}$ should equal unity.

\section{1. dAu collisions}

PHENIX measurements of $R_{d \mathrm{Au}}$ [10] provides with a means to size-up the CNM effects at play at RHIC energy. We shall compare the CNM effects obtained in the intrinsic and extrinsic schemes to these data.

Fig. 3a shows $R_{d \text { Au }}$ versus $y$. Let us first focus on the curves without nuclear absorption. The enhancement due to antishadowing is shifted to more negative $y$ in the intrinsic scheme ${ }^{5}$ compared to the extrinsic scheme. This is easily explained. We saw in section 2.4 that the typical values of $x$ are increased in the extrinsic scheme for given $y$ 's. A less

\footnotetext{
${ }^{5}$ The dot dashed curve is obtained for a fixed $\mu_{F}=M_{\psi}$ and the dashed one with open squares for $\mu_{F}=m_{T}$ using the $P_{T}$ distribution of the $p p$ data in the forward rapidity region [13]. The results are in practice identical if we consider the distribution in another $y$-region. In the following, all the curves covering the entire rapidity range for the intrinsic scheme are obtained as this dashed curve.
} 


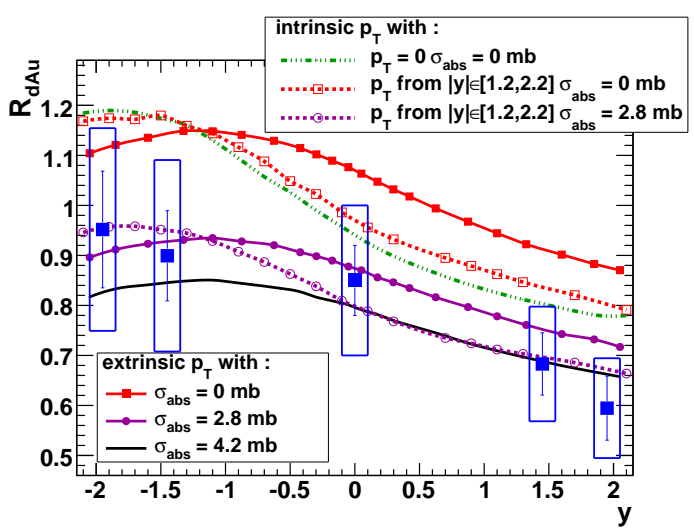

(a) $R_{d \mathrm{Au}}$ versus $y$ in the intrinsic scheme (dashed lines) and extrinsic scheme (continuous lines), for several values of the nuclear absorption cross section $\sigma_{\text {abs }}$.

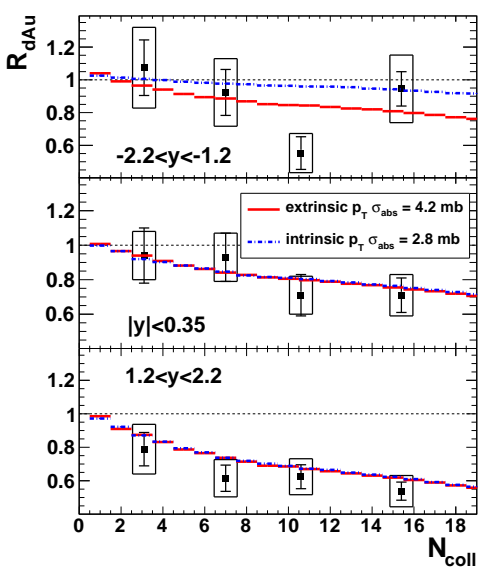

(b) $R_{d \mathrm{Au}}$ versus $N_{\text {coll }}$.

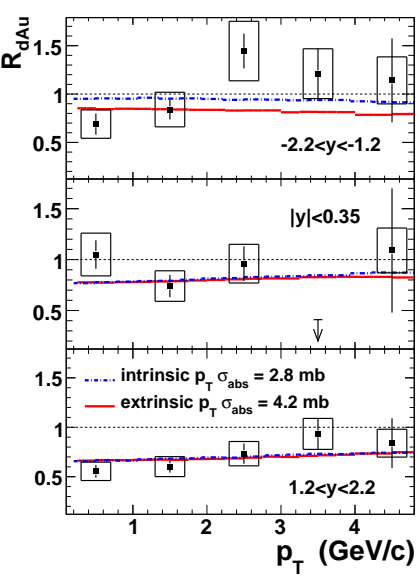

(c) $R_{d \mathrm{Au}}$ versus $P_{T}$.

Figure 3: $J / \psi$ nuclear modification factor in $d \mathrm{Au}$ collisions at $\sqrt{s_{N N}}=200 \mathrm{GeV}$.

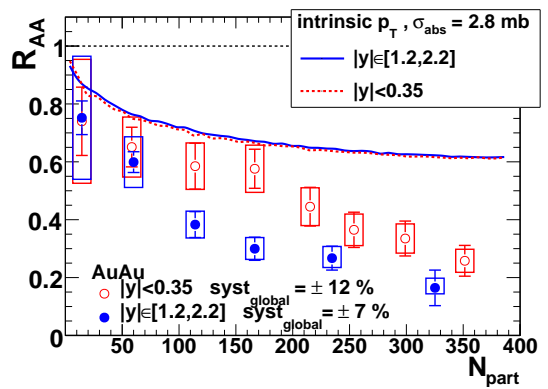

(a) $R_{\mathrm{AuAu}}$ in the intrinsic scheme.

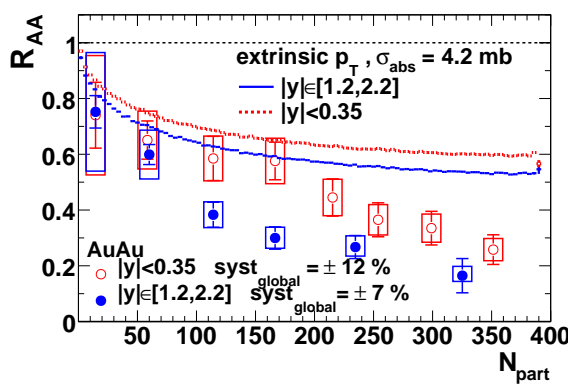

(b) $R_{\mathrm{AuAu}}$ in the extrinsic scheme.

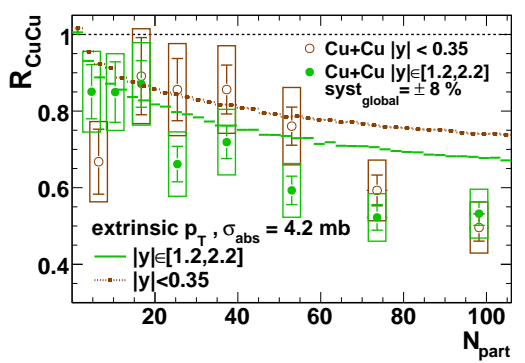

(c) $R_{\mathrm{CuCu}}$ in the extrinsic scheme.

Figure 4: $N_{\text {part }}$ dependence of the $J / \psi$ nuclear modification factor in $\mathrm{CuCu}$ and AuAu collisions at $\sqrt{s_{N N}}=200 \mathrm{GeV}$.

negative value of $y$ is therefore required to obtain a value of $x_{2}$ producing the maximum amount of antishadowing as seen on Fig. 3a.

However, as usually done, it is necessary to add the nuclear absorption. In the intrinsic scheme, we have used the value of $\sigma_{\text {abs }}=2.8 \mathrm{mb}$ from Ref. [10], where it was obtained by fitting the data with a shadowing-correction calculation equivalent to neglecting $P_{T}$ in the intrinsic scheme. A higher value of $\sigma_{\text {abs }}$ is required in the extrinsic scheme. We used $\sigma_{\mathrm{abs}}=4.2 \mathrm{mb}$ which gives a good agreement with PHENIX $d \mathrm{Au}$ data $^{6}$. The resulting curves are also plotted on Fig. 3a. From now on, we will keep these values for the value of $\sigma_{\text {abs }}$ in the respective schemes.

Fig. $3 \mathrm{~b}$ shows $R_{d \mathrm{Au}}$ as a function of $N_{\text {coll }}$ in the three rapidity windows. Both nuclear shadowing and absorption are included. Both schemes agree well with the data, especially at mid- $y$

\footnotetext{
${ }^{6} \mathrm{~A}$ complete fitting procedure taking into account the experimental errors and their correlations is beyond the scope of this first analysis of the extrinsic effects. By limiting our analysis to a constant value for $\sigma_{\mathrm{abs}}$, we also disregard possible formation-time effects.
}

Fig. 3c shows $R_{d \mathrm{Au}}$ as a function of $P_{T}$ in the three rapidity windows. The intrinsic and extrinsic approaches give similar results. They both give a reasonable agreement with the data.

\subsection{Nucleus-nucleus collisions}

We now extend our study to $A A$ collisions, where the $J / \psi$ yield may be further affected by dense matter effects. In AuAu collisions [7], PHENIX has measured an unexpected stronger $J / \psi$ suppression at forward- $y$ than at mid$y$. A possible explanation lies in the recombination scenarios [6]. In the following, we shall rather investigate if part of this rapidity-dependent suppression may be due to CNM effects. We shall also compare our results to PHENIX $\mathrm{CuCu}$ data [24]. They cover with better precision the lower $N_{\text {part }}$ range, where a smaller amount of dense matter effects is expected.

Fig. 4a shows $R_{\text {AuAu }}$ versus $N_{\text {part }}$ in the intrinsic scheme, while Fig. 4b and Fig. $4 \mathrm{c}$ show $R_{\mathrm{CuCu}}$ and $R_{\mathrm{AuAu}}$ for the extrinsic scheme. As regards $R_{\mathrm{CuCu}}$ and $R_{\mathrm{AuAu}}$ versus $y$, they are displayed on Fig. 5 for four different centrality bins. 
In the intrinsic case, $R_{\mathrm{AuAu}}$ is nearly independent of $y$ (Fig. 5 up). Indeed, for any value of $y$ in the rapidity range $-2<y<2$, the suppression at $y>0$ in one nucleus is compensated by an enhancement at $y<0$ in the other nucleus, thus giving the same result as at mid-rapidity.

On the other hand, in the extrinsic scenario, this cancellation is not as effective and both $R_{\mathrm{CuCu}}$ and $R_{\mathrm{AuAu}}$ show a maximum at $y=0$ (Fig. 5). This is explicit in Fig. $4 \mathrm{~b}$ and Fig. 4c where the curves for the central and forward rapidity ranges are shifted from each other, as the data are.
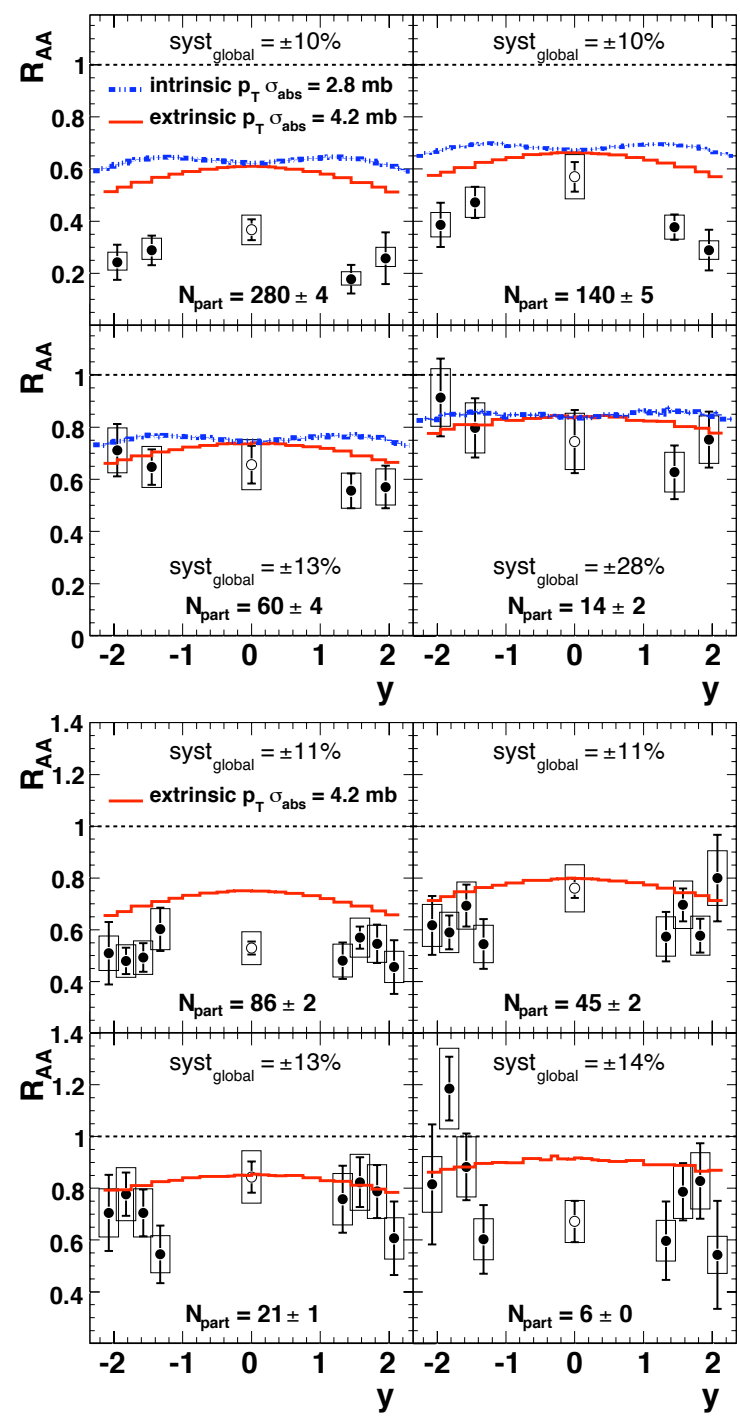

Figure 5: $J / \psi$ nuclear modification factor versus rapidity in $\mathrm{AuAu}$ (top) and $\mathrm{CuCu}$ (bottom) collisions.

\section{Conclusion and outlook}

We have evaluated Cold Nuclear Matter effects on $J / \psi$ production in proton-nucleus and nucleus-nucleus collisions at relativistic energies. We have considered the $J / \psi$ transverse momentum effects within the specific kinematics of the $J / \psi$ production at the partonic level. We have studied both intrinsic ( $2 \rightarrow 1$-like process) and extrinsic $(2 \rightarrow 2$ like process) production schemes.

We have obtained different gluon-shadowing-induced effects in $d \mathrm{Au}$ depending on the considered scheme. We have then included the nuclear absorption cross section needed to reproduce PHENIX $d \mathrm{Au}$ data [10]. In the simplified kinematics of a $2 \rightarrow 1$ process (previously considered in $[11,12,13])$, we have used $\sigma_{\text {abs }}=2.8 \mathrm{mb}$ according to [10]. Within the extrinsic scheme, a larger break-up cross section is needed and we used $\sigma_{\text {abs }}=4.2 \mathrm{mb}$.

Concerning nucleus-nucleus collisions, we have studied both $\mathrm{CuCu}$ and $\mathrm{AuAu}$ collisions in order to compare our prediction with PHENIX measurements [7, 24]. Within the extrinsic scheme, we observed a rapidity dependence of $R_{\mathrm{CuCu}}$ and $R_{\mathrm{AuAu}}$, in the same direction as the one seen in the data.

In the near future, we plan to extend our investigations to the LHC energies, where we could consider the production of $\Upsilon$ in $p A$ and $A A$ collisions at nonzero $P_{T}$ by interfacing partonic matrix elements obtained at NLO $[25,26]$ and $\mathrm{NNLO}^{\star}[27]$ with our code. We could also broaden the present study using other partonic matrix elements for $J / \psi$ production, by considering other parametrisations for the shadowing. A careful comparison with results from the Colour-Evaporation Model at NLO [28] is planned. A better treatment of the interaction between the $c \bar{c}$ pair and the nuclear matter could be also achieved by taking into account coherence effects such as the $c$-quark shadowing [29]. Studies of the $k_{T}$ broadening are also envisioned.

In summary, we have demonstrated that the kinematics of the partonic processes responsible for the $J / \psi$ production is of particular relevance to assess the importance of CNM effects both in $p A$ and $A A$ at RHIC energies. Moreover, we argue that a significant part of the rapidity dependence of $R_{A A}$ in the central collisions can be accounted by CNM effects only.

\section{Acknowledgments}

We would like to thank S. J. Brodsky, J. Cugnon, O. Drapier, R. Granier de Cassagnac, P. Hoyer, B. Kopeliovich, M. Leitch, C. Lourenço, H. J. Pirner, C. A. Salgado, J. Stachel and R. Vogt for stimulating and useful discussions. E. G. F. thanks Xunta de Galicia (2008/012) and Ministerios de Educacion y Ciencia of Spain (FPA2008-03961E/IN2P3) for financial support. This work is supported in part by a Francqui fellowship of the Belgian American Educational Foundation and by the U.S. Department of Energy under contract number DE-AC02-76SF00515.

\section{References}

[1] J. P. Lansberg, Int. J. Mod. Phys. A 21 (2006) 3857

[2] J. P. Lansberg, Eur. Phys. J. C 61 (2009) 693.

[3] R. Rapp, D. Blaschke and P. Crochet, arXiv:0807.2470 [hep-ph]

[4] J. P. Lansberg et al., AIP Conf. Proc. 1038, 15 (2008) [0807.3666 [hep-ph]]. 
[5] T. Matsui and H. Satz, Phys. Lett. B 178 (1986) 416.

[6] L. Grandchamp, R. Rapp and G. E. Brown, Phys. Rev. Lett. 92 (2004) 212301; E. L. Bratkovskaya, A. P. Kostyuk, W. Cassing and H. Stoecker, Phys. Rev. C 69 (2004) 054903; R. L. Thews, Eur. Phys. J. C 43 (2005) 97; L. Yan, P. Zhuang and N. Xu, Phys. Rev. Lett. 97 (2006) 232301; A. Andronic, P. Braun-Munzinger, K. Redlich and J. Stachel, Nucl. Phys. A 789 (2007) 334; A. Capella, L. Bravina, E. G. Ferreiro, A. B. Kaidalov, K. Tywoniuk and E. Zabrodin, arXiv:0712.4331[hep-ph].

[7] A. Adare et al., Phys. Rev. Lett. 98, 232301 (2007).

[8] A. Adare et al., Phys. Rev. Lett. 98232002 (2007).

[9] B. Alessandro et al., Eur. Phys. J. C 48 (2006) 329.

[10] A. Adare et al., Phys. Rev. C 77 (2008) 024912.

[11] T. Gousset and H. J. Pirner, Phys. Lett. B 375 (1996) 349; R. Vogt, Phys. Rev. C 61 (2000) 035203; F. Arleo and V. N. Tram, Eur. Phys. J. C 55 (2008) 449; F. Arleo, Phys. Lett. B 666 (2008) 31; H. K. Wöhri, contribution to the Hard Probe 2008 Conference, Spain, June 2008.

[12] R. Vogt, Phys. Rev. C 71 (2005) 054902.

[13] E. G. Ferreiro, F. Fleuret and A. Rakotozafindrabe, Eur. Phys. J. C 61 (2009) 859 [0801.4949 [hep-ph]]; A. Rakotozafindrabe, AIP Conf. Proc. 1038, 63 (2008) [0806.3678 [hep-ph]].

[14] J. Hufner, Y. Kurihara and H. J. Pirner, Phys. Lett. B 215 (1988) 218.

[15] H. Haberzettl and J. P. Lansberg, Phys. Rev. Lett. 100 (2008) 032006.

[16] P. Faccioli, C. Lourenco, J. Seixas and H. K. Woehri, JHEP 0810 (2008) 004.

[17] P. E. Hodgson, Nuclear Reactions and Nuclear Structure, Clarendon Press, (1971) $453 \mathrm{p}$

[18] K. J. Eskola, V. J. Kolhinen and C. A. Salgado, Eur. Phys. J. C 9 (1999) 61.

[19] D. de Florian and R. Sassot, Phys. Rev. D 69 (2004) 074028.

[20] K. J. Eskola, H. Paukkunen and C. A. Salgado, JHEP 0807 (2008) 102.

[21] S. R. Klein and R. Vogt, Phys. Rev. Lett. 91 (2003) 142301.

[22] J. P. Lansberg, J. R. Cudell and Yu. L. Kalinovsky, Phys. Lett. B 633 (2006) 301

[23] C-H. Chang, Nucl. Phys. B 172 (1980) 425; R. Baier and R. Rückl, Phys. Lett. B 102 (1981) 364; R. Baier and R. Rückl, Z. Phys. C 19 (1983) 251.

[24] A. Adare et al., Phys. Rev. Lett. 101 (2008) 122301 ; S. Odaet al., J. Phys. G 35, 104134 (2008).

[25] J. Campbell, F. Maltoni and F. Tramontano, Phys. Rev. Lett. 98 (2007) 252002.

[26] P. Artoisenet, J. P. Lansberg and F. Maltoni, Phys. Lett. B 653 (2007) 60.

[27] P. Artoisenet, J. Campbell, J. P. Lansberg, F. Maltoni and F. Tramontano, Phys. Rev. Lett. 101, 152001 (2008), [0806.3282 [hep-ph]].

[28] M. Bedjidian et al., CERN-2004-009-C, arXiv:hep-ph/0311048.

[29] B. Kopeliovich, A. Tarasov and J. Hufner, Nucl. Phys. A 696 (2001) 669. 